\title{
Toxicodynamic Effects of 'Red Bull' Energy Drink in A Randomised Controlled Study on Local Strains of Adult Rabbits
}

Taiwo, Oluwakemi Iyaniwura (Corresponding author) \& Adesokan, Ayoade Abdulfatai

Department of Biochemistry, Faculty of Basic Medical Sciences,

Ladoke Akintola University of Technology, PMB 4000, Ogbomoso,

Nigeria. E-mail: kisstaiwo1@gmail.com

Received: January 17, 2018 Accepted: February 1, 2018

doi:10.5296/jbls.v9i1.12651ＵRL: https://doi.org/10.5296/jbls.v9i1.12651

\begin{abstract}
Increasing reports of anecdotal cases of acute adverse effects associated with the consumption of energy drink have been expressed by international bodies. This has necessitated the need to assess toxicological effects of Red Bull (RB) energy drink in this study. Eighteen adult rabbits were randomly divided into 3 groups of 6 rabbits (3 males and 3 females). Rabbits in groups 1, 2 and 3 (control) received daily oral doses of $23.1 \mathrm{ml} \mathrm{RB}, 11.5$ $\mathrm{ml} \mathrm{RB}$ and water respectively for 21 days. Venous samples were collected on days 1 (baseline), 14, 21 and 28, and animals were sacrificed on days 21 and 28. Biochemical assessment of liver and renal functions, and muscle activity were carried out by spectrophotometry, in addition to haematological and histological studies. Mortality of $11.1 \%$ was recorded, involving 2 female rabbits in group 1. For male rabbits in this group, significant increase $(\mathrm{p}<0.05)$ in total protein $(\mathrm{TP})$ concentration and in activity of ALT and ALP occurred on days 14 and 21, with significant decrease in platelet counts. For female rabbits in group 2, significant increases in TP concentration and ALT activity were observed with significant decrease in albumin concentration and AST activity. Apart from slight increase in ALP activity of female rabbits, there were no significant differences in mean values of all rabbit parameters measured in the control group. Histology of rabbits in group 1 showed congestion and balloon degeneration of hepatocytes. This result showed that administration of $\mathrm{RB}$ to rabbits at doses lower than the manufacturer's recommended equivalent daily human intake of $500 \mathrm{ml}$ caused adverse effects. There is need for caution in consumption of this drink.
\end{abstract}

Keywords: 'energy drink', toxicodynamic, rabbit, histology, adverse effect 


\section{Introduction}

The term "energy drinks" has been used to encompass a category of beverages that have appeared in the European, North American and African markets in recent years. These drinks contain stimulating substances such as caffeine, guarana, ginseng or other plant extracts, in combinations with other substances such as carbohydrates, vitamins and minerals. They should however not be confused with 'sports drinks' or 'energetic drinks' which are typically mixture of carbohydrates and electrolytes formulated to enhance athletic performance and prevent dehydration, but do not contain stimulants (Rotstein et al., 2013).

The use of the term "energy drinks" is not an agreed legal term for a category of foods but more of a commercial designation (Stimulant Drink Committee, 2002). Most health or food regulatory authorities of countries do not have a definition or standard for products known as energy drinks, although some food regulators have reached a decision on this aspect of the products (Rotstein et al., 2013). For example, countries like Australia and New Zealand, have categorized energy drinks as "formulated caffeinated beverages" which are defined under the Australian New Zealand Food Standards Code as "non-alcoholic water-based flavored beverage that contain caffeine and may contain carbohydrates, amino acids, vitamins and other substances, including other foods, for the purpose of enhancing mental performance" (ANZFSC, 2000). The expert committee established by the Ireland Food Safety Promotion Board (FSPB) adopted the term "stimulant drinks" for energy drinks and defined it as "beverages which typically contain caffeine, taurine and vitamin(s), and may contain an energy source (e.g. carbohydrates), and/or other substance(s), marketed for the specific purpose of providing real or perceived enhanced physiological and/or performance effects" (FSPB, 2003).

However, concerns have been raised about energy drinks in response to anecdotal cases of acute adverse effects requiring medical attention in young people consuming energy drinks, in most cases, in conjunction with alcohol and/or drugs used socially. Such effects include tremors, seizures, drowsiness, muscle weakness, dizziness, nervousness, tachycardia, palpitations, nausea, vomiting, headache, bronchospasm and hyperventilation (SCF, 2003). One case of myocardial infarction in a 23 year old playing football (Rallis, 2001) and one case of sudden unexplained adult death syndrome, possibly resulting from cardiac dysrhythymia, in an 18 year old playing basketball after drinking 4 cans of RB energy drink (Stimulant Drinks Committee, 2002) have also been reported. There is however no confirmation of any causal relationship between these reported effects and the consumption of energy drinks.

The first and the best known energy drinks is Red Bull, developed in Austria in 1987 (Red Bull $\left.^{(\mathrm{R})} \mathrm{GmbH}\right)$. The components of this drink included B vitamins, glucuronolactone, sodium, caffeine and synthetically derived amino acid, taurine. The Red Bull selling proposition was that it increased stamina and mental concentration making it natural for one of the original target users, the long-distance drivers. Red Bull's distribution from Austria expanded into neighboring countries such as Hungary and Slovenia in 1992, Germany and United Kingdom in 1994, United States in 1997, South Africa and Brazil in 2001, United Arab Emirates in 


\section{Macrothink}

2002, and Canada in 2004. The exact time Red Bull drink entered Nigeria is not clear but there are several other energy drink brands available in the country. There has been very little research in Nigeria (Akande and Banjoko, 2011; Ebuehi et al., 2011; Chimezie, 2013) examining energy drinks performance, contents, consumption rate or side effects in laboratory experimental animal or human studies.

It is postulated that some of the adverse effects reported from consumption of energy drinks may be due to yet unknown interactions between caffeine, taurine and other contents of the drink, rather than known individual effects of each ingredient. The aim of this study is to assess the sub-acute toxicodynamic effects of oral Red Bull (RB) 'energy' drink on biochemical, haematological and histologic parameters of local strains of rabbits, in order to establish the safety of these 'energy' drinks for human consumption.

\section{Materials and method}

\section{Study area}

This study was conducted at the Department of Biochemistry, College of Health Sciences, Ladoke Akintola University of Technology (LAUTECH), Ogbomoso, Nigeria.

\section{Study design}

The research is a randomised controlled toxicodynamic effect of repeated dose (28-day) Red Bull 'energy' drinks on biochemical, haematological and histologic parameters of adult rabbits.

\section{Reagents and consumables}

A pack of Red Bull(R) energy drink (GmbH; A-5330 Fuschl am See/Austria; Batch No 136489) containing 24 cans (each can contain $250 \mathrm{ml}$ of fluid) was purchased at local supermarket in Osogbo, Nigeria. A $250 \mathrm{ml}$ can of RB contained $1000 \mathrm{mg}$ of taurine and 80 $\mathrm{mg}$ of caffeine in addition to other constituents. Drinks were preserved at room temperature in accordance with manufacturers' specifications. Analytical grade commercially prepared biochemical reagents were purchased from Randox Diagnostics, United Kingdom and kept frozen at $-20 \mathrm{oC}$ until use

\section{Experimental and control rabbits}

Local strains of apparently healthy adult rabbits (a total of 18, 9 males and 9 females) with average weight of $1.8 \mathrm{~kg}$ obtained from the Animal House of the College of Health Sciences, LAUTECH, Osogbo, were used in the study. All rabbits were housed in metal cages of maximum of 2 rabbits (of the same sex)/cage in a well-ventilated experimental room to acclimatize for 7 days at temperature of 20-26 oC, relative humidity of 40-60\% and exposure to $12 \mathrm{~h}$ light and dark cycles. The study was performed in accordance with Good Laboratory Practice (GLP) regulations and the ethical guidelines of the Organization for Economic Cooperation and Development (OECD, 2008) for the use of laboratory animals

The rabbits were randomly divided into 3 groups of 6 rabbits each ( 3 males and 3 females in each group) after 7 days of acclimatization. Rabbits in groups 1 and 2 represented the 
experiment group while those in group 3 served as control. Rabbits in each group were fed the same standard laboratory diet and water ad libitum throughout the period of the experiment.

\section{Study protocol}

The volumes of RB drink administered to rabbits in test groups 1 and 2 was calculated using the animal to human equivalent dose (and vice versa) conversion formula based on surface area (USFDA, 2005; Shin et al., 2010). Based on this formula, rabbits in group 1 received (in addition to normal feeds) $23.1 \mathrm{ml} \mathrm{RB}$ energy drink (maximum tolerable dose) by oral drenching with the aid of a metal cannula daily for 21 days (equivalent of $250 \mathrm{ml}$ or one can of $\mathrm{RB}$ drink daily intake in a $60 \mathrm{~kg}$ adult) while rabbits in group 2 received $11.5 \mathrm{ml}$ of the drink (equivalent of $125 \mathrm{ml}$ or half can). Rabbits in group 3 received normal standard laboratory diet for 28 days.

\section{Observation of behavioural changes}

Changes in the behaviour of animals in the test and control groups were noted by daily observation of physical or mental status before and 1 hour after administration of the substance.

\section{Blood sample collection for biochemical and haematological analyses}

Approximately 5 milliliters of blood were collected from the ear lobe vein of each rabbit in all three groups using sterile needle and syringe early in the morning, two hours before feeding and administration of energy drink. Samples were collected on day 1 (for evaluation of baseline parameters) and on days 14, 21 and 28. The blood samples were collected into Lithium Heparin bottles for biochemical analysis and Ethylene Diamine Tetra acetic acid (EDTA) bottles for haematological parameters. Samples for biochemical analysis were first centrifuged at $5000 \mathrm{x}$ g for $3 \mathrm{~min}$ in a bucket centrifuge (Model $800 \mathrm{D} \mathrm{CE}$ ) to separate plasma which was then pipetted into separate plain bottles, and kept frozen at $-20 \mathrm{oC}$ until time of analysis.

\section{Tissue collection for histopathology}

On day 21, test substances was withdrawn from all rabbits in the test groups, and two rabbits ( 1 male and 1 female) in each of the test groups and 2 rabbits in the control group were sacrificed by cervical dislocation. All other rabbits were sacrificed on day 28. Relevant organs (liver, lung, kidney, heart, stomach and intestine) were harvested and fixed with neutral buffer $10 \%$ formalin for histopathological analysis.

\section{Biochemical assessment of liver function}

Liver functions were assessed by testing for plasma proteins (total protein and albumin) and liver enzymes (Aspartate Transaminase AST, Alanine Transaminase ALT and Alkaline Phosphatase ALP). Total plasma protein was estimated using Biuret method that is based on protein forming a complex with cupric ion in alkaline solution to produce color reaction that is measured at $540 \mathrm{~nm}$ with yellow green filter in comparison to standard plasma of known 
protein concentration (Reinhold, 1953).

Plasma albumin was measured using routine method of Tietz (2000). The kinetic determination of ALT activity was according to the coupling reaction involving pyridoxal-5'-phophate and lactate dehydrogenase at $37{ }^{\circ} \mathrm{C}$ with decrease in absorbance measured at $340 \mathrm{~nm}$ by continuous monitoring while that of AST activity was based on coupling reaction involving pyridoxal-5'-phophate and malate dehydrogenase at $37{ }^{\circ} \mathrm{C}$ with decrease in absorbance measured at $340 \mathrm{~nm}$ by continuous monitoring (Arneson and Brickell, 2007). Alkaline Phosphatase (ALP) activity was measured by the modified method of Bowers and McComb (1966).

\section{Biochemical assessment of renal function}

Assessment of renal functions was done by measuring plasma creatinine, urea and electrolytes (sodium, potassium, chloride and bicarbonate ions). Creatinine in plasma was estimated by the Kinetic Jaffe reaction (Arneson \& Brickell, 2007). Urea was estimated in the plasma by the colorimetric Urease-Berthelot method (Searcy et al., 1967). Plasma concentrations of sodium, potassium, chloride and $\mathrm{HCO}_{3}{ }^{-}$were measured using Ion Selective Electrode (ISE, 6000 analyzer, SFRI)

\section{Biochemical assessment of muscle activity}

Creatine kinase activity in the blood, as a function of muscle activity, was based on the reaction that $\mathrm{CK}$ catalyzes the transfer of a phosphate group from the creatine phosphate substrate to adenosine diphosphate (ADP). The subsequent formation of adenosine triphosphate (ATP) was measured through the use of two coupled reactions catalyzed by hexokinase $(\mathrm{HK})$ and glucose-6-phosphate dehydrogenase (G6PD) which results in the production of $\beta$-Nicotinamide Adenine Dinucleotide (reduced form) (NADH) from $\beta$-Nicotinamide-Adenine Dinucleotide (NAD), measured by decreased absorbance at $340 \mathrm{~nm}$ (Arneson and Brickell, 2007).

\section{Haematological analysis}

The automated haematology analyzer (Shanghai Utrao Medical Equipment Company, Model SH800Plus, China) was used to analyze complete blood count (CBC) and different leukocyte count (DLC) with the estimation of the following parameters; red blood cell (RBC) count, haemoglobin concentration, packed cell volume (PCV), white blood cell (WBC) count, platelets count and red cell indices (mean corpuscular volume (MCV), mean corpuscular haemoglobin $(\mathrm{MCH})$, mean corpuscular haemoglobin concentration $(\mathrm{MCHC})$ and red blood cell distribution width (RWD).

\section{Histopathological assessment}

Histopathological processing of organ specimens and examination of slides were done at the Histopathology Laboratory of the LAUTECH Teaching Hospital, Ogbomoso, Nigeria with the help of a Histopathologist. The fixed organs were dehydrated in ascending grades of alcohol, cleared and embedded in paraffin blocks. Sections (about 4-6 $\mu$ thickness) of paraffin-embedded tissue were cut using microtome and slides prepared and stained with 


\section{Macrothink}

Journal of Biology and Life Science ISSN 2157-6076 2018, Vol. 9, No. 1

Haematoxylin and Eosin $(H \& E)$. The stained sections were then examined under compound light microscope at $1000 \mathrm{x}$ magnification for general histological features (Luna, 1968). Photographs were taken with a camera.

\section{Statistical analysis}

Data were calculated and reported as mean \pm standard error of mean (SEM) for both male and females in each group $(n=3)$. Statistical comparisons were done between and among groups. Comparison of baseline data (day 1) with day 14 and 21 for experimental groups was performed using one way analysis of variance (ANOVA) while comparison of day 21 data with day 28 was done using Students " $t$ " test. Significance level was fixed at $p<0.05$.

\section{Result}

\section{Mortality}

Mortality was recorded in 2 rabbits in group 1 (both females) on days 4 and 6 of the study. No death was recorded in groups 2 and 3.

\section{Effects of RB drink on behaviour of rabbits in the test groups}

Rabbits in group 1 and 2 showed consistently increased activity 1 hour after oral intake of RB drink compared to control rabbits

\section{Effects of $R B$ drink on plasma proteins, electrolytes, urea and creatinine}

As shown in Table 1, the only significant effects were increase in total proteins for male rabbits in group 1 that received maximum tolerable dose $(23.1 \mathrm{ml})$ of energy drink and female rabbits in group 2 that received half $(11.5 \mathrm{ml}$ ) on day 14 and 21 (which reverted on day 28 after withdrawal of drink). However reversible reduction in albumin concentration was noticed in the female rabbits in group 2. All parameters for male rabbits in group 2 were unaffected while those of female rabbits in group 1 could not be assessed on day 14 forward as two of the three rabbits died in the first week of the study.

Table 1: Effects of 'Red Bull' energy drinks on plasma proteins, electrolytes, urea and creatinine of adult rabbits

\begin{tabular}{cccccccc}
$\begin{array}{c}\text { Parameters } \\
\text { (unit) }\end{array}$ & Days & \multicolumn{2}{c}{ Group 1 } & \multicolumn{2}{c}{ Group 2 } & \multicolumn{2}{c}{ Group 3 (control) } \\
$\mathrm{Na}^{+}$ & 1 & $135.20 \pm 2.06$ & $145.60 \pm 5.47$ & $139.43 \pm 3.37$ & $143.50 \pm 5.95$ & $137.00 \pm 7.94$ & $142.27 \pm 5.80$ \\
$(\mathrm{mmol} / \mathrm{l})$ & 14 & $158.40 \pm 11.25$ & NA & $145.60 \pm 5.47$ & $133.47 \pm 2.22$ & $135.40 \pm 11.67$ & $149.07 \pm 10.31$ \\
& 21 & $136.20 \pm 0.01$ & NA & $149.60 \pm 7.28$ & $132.70 \pm 0.81$ & $136.30 \pm 5.18$ & $145.17 \pm 1.65$ \\
& 28 & $134.60 \pm 0.10$ & NA & $142.37 \pm 10.59$ & $183.37 \pm 11.52$ & $136.27 \pm 13.57$ & $143.10 \pm 5.08$ \\
& & & & & & & \\
$\mathrm{~K}^{+}$ & 1 & $4.77 \pm 0.33$ & $4.60 \pm 0.52$ & $4.69 \pm 0.23$ & $4.59 \pm 0.44$ & $4.65 \pm 0.29$ & $4.66 \pm 0.21$ \\
$(\mathrm{mmol} / \mathrm{l})$ & 14 & $5.09 \pm 0.51$ & NA & $4.60 \pm 0.52$ & $4.17 \pm 0.06$ & $4.59 \pm 0.45$ & $5.04 \pm 0.40$ \\
& 21 & $3.26 \pm 0.00$ & NA & $4.19 \pm 0.10$ & $3.69 \pm 0.05$ & $4.50 \pm 0.45$ & $4.70 \pm 0.47$ \\
& 28 & $4.43 \pm 0.01$ & NA & $4.16 \pm 0.25$ & $4.63 \pm 0.00$ & $4.00 \pm 0.16$ & $4.30 \pm 0.21$
\end{tabular}




$\begin{array}{ccllllll}\mathrm{Cl}^{-} & 1 & 105.90 \pm 3.36 & 106.77 \pm 5.24 & 104.60 \pm 2.11 & 103.97 \pm 4.12 & 100.30 \pm 3.24 & 101.37 \pm 5.84 \\ (\mathrm{mmol} / \mathrm{l}) & 14 & 116.23 \pm 7.15 & \text { NA } & 106.77 \pm 5.24 & 96.07 \pm 0.89 & 101.10 \pm 8.64 & 113.27 \pm 3.96 \\ & 21 & 99.30 \pm 0.00 & \text { NA } & 92.30 \pm 4.45 & 98.30 \pm 1.39 & 101.40 \pm 3.24 & 107.30 \pm 1.56 \\ & 28 & 95.10 \pm 0.01 & \text { NA } & 98.80 \pm 8.98 & 71.20 \pm 0.00 & 100.30 \pm 1.44 & 102.17 \pm 3.09 \\ & & & & & & & \\ \mathrm{HCO}_{3}{ }^{-} & 1 & 7.75 \pm 0.07 & 7.70 \pm 0.04 & 7.61 \pm 0.08 & 7.73 \pm 0.10 & 7.90 \pm 0.14 & 7.75 \pm 0.14 \\ (\mathrm{mmol} / \mathrm{l}) & 14 & 7.46 \pm 0.09 & \text { NA } & 7.70 \pm 0.04 & 7.91 \pm 0.01 & 7.50 \pm 0.13 & 7.70 \pm 0.20 \\ & 21 & 7.84 \pm 0.01 & \text { NA } & 7.71 \pm 0.04 & 7.89 \pm 0.11 & 7.70 \pm 0.15 & 7.90 \pm 0.04 \\ & 28 & 7.97 \pm 0.00 & \text { NA } & 7.77 \pm 0.05 & 7.91 \pm 0.00 & 7.86 \pm 0.03 & 7.89 \pm 0.03 \\ & & & & & & & \\ \text { Urea } & 1 & 3.73 \pm 1.14 & 5.73 \pm 0.27 & 5.37 \pm 0.98 & 6.60 \pm 0.53 & 6.13 \pm 0.73 & 6.33 \pm 0.23 \\ (\mathrm{mmol} / \mathrm{l}) & 14 & 4.77 \pm 0.72 & \text { NA } & 5.73 \pm 0.27 & 7.30 \pm 0.17 & 4.70 \pm 0.31 & 4.93 \pm 0.29 \\ & 21 & 4.30 \pm 0.00 & \text { NA } & 4.60 \pm 0.23 & 5.37 \pm 0.03 & 5.13 \pm 0.52 & 5.87 \pm 0.09 \\ & 28 & 3.50 \pm 0.01 & \text { NA } & 6.03 \pm 0.78 & 5.20 \pm 0.00 & 4.93 \pm 0.03 & 4.77 \pm 0.66 \\ & & & & & & & \\ \mathrm{Creatinine} & 1 & 135.73 \pm 7.17 & 157.27 \pm 18.41 & 145.47 \pm 14.37 & 130.47 \pm 6.91 & 144.63 \pm 11.79 & 144.10 \pm 3.35 \\ (\mu \mathrm{mol} / \mathrm{l}) & 14 & 185.33 \pm 13.03 & \text { NA } & 157.27 \pm 18.41 & 159.77 \pm 13.02 & 168.97 \pm 6.19 & 168.17 \pm 7.92 \\ & 21 & 128.80 \pm 0.00 & \text { NA } & 157.20 \pm 3.87 & 121.27 \pm 4.36 & 152.70 \pm 4.69 & 139.27 \pm 6.12 \\ & 28 & 130.50 \pm 0.01 & \text { NA } & 145.50 \pm 18.43 & 127.93 \pm 1.47 & 160.60 \pm 7.74 & 136.30 \pm 0.13 \\ & & & & & & & \\ \text { TP } & 1 & 5.93 \pm 2.35 & 7.41 \pm 4.55 & 7.79 \pm 7.60 & 7.46 \pm 3.63 & 6.77 \pm 4.41 & 7.69 \pm 3.19 \\ (\mathrm{~g} / \mathrm{dl}) & 14 & 7.61 \pm 5.81^{\mathrm{a}} & \text { NA } & 7.51 \pm 4.55 & 11.29 \pm 1.66^{\mathrm{a}} & 6.59 \pm 6.52 & 7.29 \pm 5.08 \\ & 21 & 6.97 \pm 0.00^{\mathrm{a}} & \text { NA } & 7.48 \pm 6.07 & 9.23 \pm 12.07^{\mathrm{a}} & 6.80 \pm 0.00 & 7.49 \pm 8.96 \\ & 28 & 5.95 \pm 0.01^{\mathrm{d}} & \text { NA } & 7.04 \pm 8.36 & 6.82 \pm 7.79^{\mathrm{d}} & 6.86 \pm 1.65 & 8.01 \pm 5.87 \\ & 14 & 4.49 \pm 0.74 & \text { NA } & 4.54 \pm 0.81 & 4.32 \pm 0.18^{\mathrm{c}} & 4.46 \pm 0.93 & 4.63 \pm 0.05 \\ & 21 & 4.29 \pm 0.01 & \text { NA } & 4.56 \pm 1.13 & 4.27 \pm 0.11^{\mathrm{c}} & 4.51 \pm 0.00 & 4.57 \pm 0.64 \\ & & 4.51 \pm 0.01 & \text { NA } & 4.37 \pm 0.90 & 4.65 \pm 0.55^{\mathrm{b}} & 4.45 \pm 0.49 & 4.53 \pm 0.91\end{array}$

Data represented as mean \pm SEM ( $\mathrm{n}=3$ for day 1,14 and 21 ; $\mathrm{n}=2$ for day 28 ), NA = mean value not available as 2 of the 3 female rabbits in group1 died within a week of the study, superscript a = significant increase on days 14 and 21 over day 1 ( $p<0.05$ by ANOVA), superscript $b=$ significant increase on day 28 over day 21 ( $p<0.05$ by Student's $t$ test), superscript $\mathrm{c}=$ significant decrease on days 14 and 21 over day 1 ( $p<0.05$ by ANOVA), superscript $d=$ significant decrease on day 28 over day 21 ( $\mathrm{p}<0.05$ by Student's $\mathrm{t}$ test $), \mathrm{TP}=$ Total Protein, $\mathrm{Na}^{+}=$Sodium ion, $\mathrm{K}^{+}=$Potassium ion, $\mathrm{M}=$ Male, $\mathrm{F}=$ Female, SEM = Standard Error of Mean 


\section{MInstitute"}

\section{Effects of Red Bull drink on plasma liver and muscle enzyme activities}

Table 2 \& Fig 1 shows reversible significant increase in ALT and non-reversible increase in ALP activities (Fig 2) in group 1 male rabbits but decrease in AST activity. ALT activity was also significantly increased in group 2 female rabbits which reversed upon withdrawal of the energy drink on day 21. The mean AST activity also decreased but ALP activity was unaffected. Apart from slight increase in mean activity of ALP in female rabbits in group 3 (control), the mean values of all other parameters of the control rabbits are not significantly different on days $1,14,21$ and 28 .

Table 2: Effects of 'Red Bull' energy drinks on liver and muscle enzymes activities of adult rabbits

\begin{tabular}{ccllllll}
$\begin{array}{c}\text { Parameters } \\
\text { (unit) }\end{array}$ & Days & \multicolumn{1}{c}{ Group 1 } & \multicolumn{2}{c}{ Group 2 } & \multicolumn{2}{c}{ Group 3 (control) } \\
AST & 1 & $144.80 \pm 33.72$ & $125.38 \pm 9.98$ & $144.34 \pm 7.94$ & $137.28 \pm 5.33$ & $74.84 \pm 7.65$ & $90.28 \pm 6.40$ \\
$(\mathrm{u} / \mathrm{l})$ & 14 & $95.95 \pm 34.44$ & NA & $125.38 \pm 9.98$ & $49.04 \pm 6.48^{\mathrm{c}}$ & $91.63 \pm 10.32$ & $85.74 \pm 1.79$ \\
& 21 & $93.42 \pm 0.00$ & NA & $136.51 \pm 13.72$ & $37.30 \pm 13.27^{\mathrm{c}}$ & $101.30 \pm 0.33$ & $94.97 \pm 5.75$ \\
& 28 & $40.07 \pm 0.10^{\mathrm{d}}$ & NA & $155.03 \pm 10.92$ & $48.27 \pm 8.21$ & $77.19 \pm 1.72$ & $100.60 \pm 5.98$ \\
& & & & & & & \\
ALT & 1 & $30.67 \pm 8.69$ & $24.67 \pm 3.89$ & $36.67 \pm 2.96$ & $21.77 \pm 1.67$ & $32.50 \pm 15.46$ & $37.83 \pm 9.73$ \\
$(\mathrm{u} / \mathrm{l})$ & 14 & $46.00 \pm 10.54^{\mathrm{a}}$ & NA & $34.67 \pm 3.89$ & $75.50 \pm 0.29^{\mathrm{a}}$ & $44.00 \pm 14.05$ & $88.77 \pm 12.27$ \\
& 21 & $74.00 \pm 0.01^{\mathrm{a}}$ & NA & $60.75 \pm 19.77$ & $66.50 \pm 4.91^{\mathrm{a}}$ & $43.00 \pm 0.00$ & $84.00 \pm 19.63$ \\
& 28 & $58.00 \pm 0.10^{\mathrm{d}}$ & NA & $44.30 \pm 17.61$ & $32.00 \pm 10.39^{\mathrm{d}}$ & $58.27 \pm 1.01$ & $31.00 \pm 2.59$ \\
& & & & & & & \\
ALP & 1 & $56.00 \pm 10.69$ & $49.67 \pm 4.91$ & $49.67 \pm 8.51$ & $61.33 \pm 19.62$ & $61.33 \pm 29.85$ & $59.70 \pm 15.89$ \\
$(\mathrm{u} / \mathrm{l})$ & 14 & $70.00 \pm 12.12^{\mathrm{a}}$ & NA & $49.67 \pm 4.91$ & $85.50 \pm 15.88$ & $126.67 \pm 39.18$ & $111.50 \pm 18.19^{\mathrm{a}}$ \\
& 21 & $129.00 \pm 0.01^{\mathrm{a}}$ & NA & $71.50 \pm 3.18$ & $204.00 \pm 52.54$ & $106.67 \pm 39.18$ & $121.00 \pm 6.35^{\mathrm{a}}$ \\
& 28 & $171.00 \pm 0.01^{\mathrm{b}}$ & NA & $114.70 \pm 32.05$ & $123.00 \pm 27.71$ & $103.67 \pm 0.33$ & $136.50 \pm 7.22$ \\
& & & & & & & \\
CK & 1 & $286.87 \pm 72.32$ & $396.63 \pm 98.17$ & $295.05 \pm 44.08$ & $372.93 \pm 138.83$ & $311.00 \pm 127.71$ & $288.00 \pm 72.68$ \\
$(\mathrm{u} / \mathrm{l})$ & 14 & $295.43 \pm 7.79$ & NA & $306.63 \pm 90.17$ & $192.00 \pm 1.16$ & $242.40 \pm 8.89$ & $338.10 \pm 161.40$ \\
& 21 & $215.70 \pm 0.01$ & NA & $449.87 \pm 47.66$ & $511.77 \pm 57.19$ & $226.47 \pm 2.05$ & $265.17 \pm 47.52$ \\
& 28 & $132.40 \pm 0.01^{\mathrm{d}}$ & NA & $288.9 \pm 165.41$ & $113.50 \pm 29.79$ & $237.30 \pm 3.58$ & $160.97 \pm 23.82$
\end{tabular}

Data represented as mean \pm SEM ( $\mathrm{n}=3$ for day 1,14 and $21 ; \mathrm{n}=2$ for day 28 ), $\mathrm{NA}=$ mean value not available as 2 of the 3 female rabbits in group1 died within a week of the study, superscript a = significant increase on days 14 and 21 over day 1 ( $p<0.05$ by ANOVA), superscript $b=$ significant increase on day 28 over day 21 ( $\mathrm{p}<0.05$ by Student's $\mathrm{t}$ test), superscript $\mathrm{c}=$ significant decrease on days 14 and 21 over day 1 ( $p<0.05$ by ANOVA), superscript $d=$ significant decrease on day 28 over day 21 ( $\mathrm{p}<0.05$ by Student's t test), ALT = Alanine transaminase, AST = Aspartate transaminase, $\mathrm{ALP}=$ Alkaline phosphatase, $\mathrm{CK}=$ Creatine kinase, $\mathrm{M}=$ Male, $\mathrm{F}=$ Female, $\mathrm{SEM}=$ Standard Error of Mean 


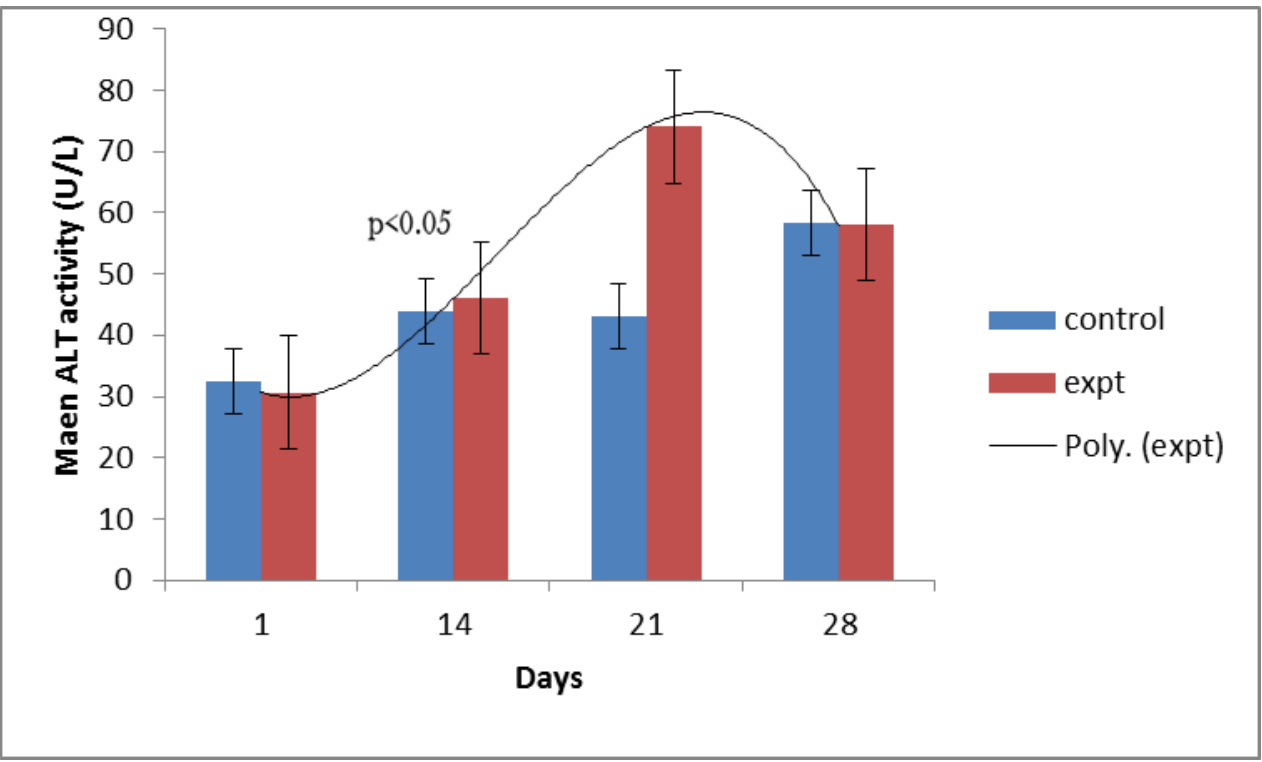

Fig 1: Effects of $23.1 \mathrm{ml} \mathrm{RB}$ drink on ALT activity of male rabbits in group 1

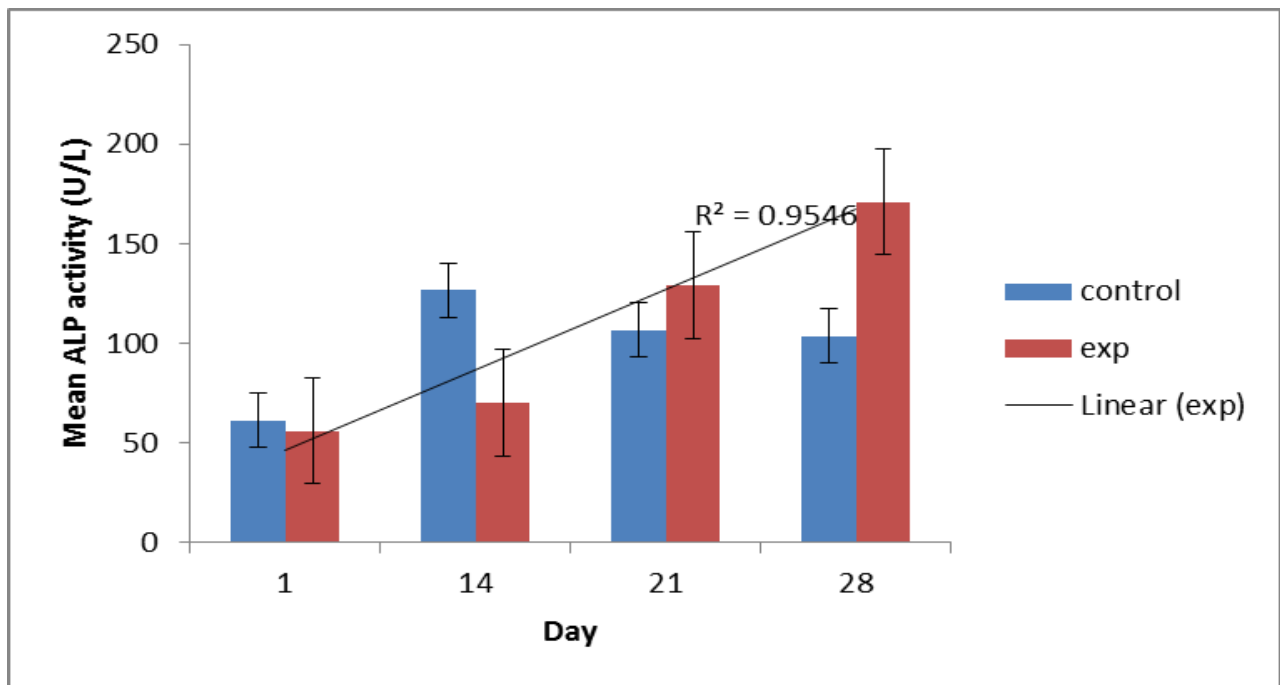

Fig 2: Effects of $23.1 \mathrm{ml} \mathrm{RB}$ drink on ALP activity of male rabbits in group 1

\section{Effects of Red Bull on lipid profile and haematological parameters of rabbits}

In Table 3, the only significant effects are reversible increase in LDL cholesterol concentration in male rabbits in group 1 and significant reduction in platelet concentration (Fig 3). All parameters of rabbits in group 2 are not significantly affected. 


\section{A Macrothink}

Journal of Biology and Life Science

ISSN 2157-6076

2018, Vol. 9, No. 1

Table 3: Effects of 'Red Bull' energy drinks on lipid profile and haematological parameters of adult rabbits

\begin{tabular}{|c|c|c|c|c|c|c|c|}
\hline \multirow{2}{*}{$\begin{array}{l}\text { Parameters } \\
\text { (units) }\end{array}$} & \multirow[t]{2}{*}{ Days } & \multicolumn{2}{|c|}{ Group 1} & \multicolumn{2}{|c|}{ Group 2} & \multicolumn{2}{|c|}{ Group 3 (control) } \\
\hline & & M & $\mathrm{F}$ & M & $\mathrm{F}$ & M & $\mathrm{F}$ \\
\hline \multirow{4}{*}{$\begin{array}{c}\mathrm{TC} \\
(\mathrm{mmol} / \mathrm{l})\end{array}$} & 1 & $1.86 \pm 0.37$ & $2.70 \pm 0.13$ & $1.18 \pm 0.09$ & $2.27 \pm 0.48$ & $1.46 \pm 0.45$ & $2.47 \pm 0.87$ \\
\hline & 14 & $1.46 \pm 0.29$ & NA & $1.17 \pm 0.13$ & $4.34 \pm 0.00$ & $2.39 \pm 0.73$ & $2.21 \pm 0.23$ \\
\hline & 21 & $2.31 \pm 0.01$ & NA & $1.12 \pm 0.22$ & $2.87 \pm 0.84$ & $1.28 \pm 0.04$ & $2.19 \pm 0.18$ \\
\hline & 28 & $0.91 \pm 0.00$ & NA & $1.61 \pm 0.27$ & $5.15 \pm 0.00$ & $0.81 \pm 0.05$ & $1.74 \pm 0.48$ \\
\hline \multirow{4}{*}{$\begin{array}{c}\text { TG } \\
(\mathrm{mmol} / \mathrm{l})\end{array}$} & 1 & $0.42 \pm 0.29$ & $1.03 \pm 0.31$ & $0.61 \pm 0.14$ & $0.34 \pm 0.09$ & $0.21 \pm 0.08$ & $0.23 \pm 0.09$ \\
\hline & 14 & $0.18 \pm 0.12$ & NA & $1.03 \pm 0.31$ & $0.34 \pm 0.00$ & $0.17 \pm 0.04$ & $0.73 \pm 0.36$ \\
\hline & 21 & $0.42 \pm 0.01$ & NA & $1.39 \pm 0.29$ & $0.33 \pm 0.01$ & $0.38 \pm 0.03$ & $0.29 \pm 0.11$ \\
\hline & 28 & $0.18 \pm 0.00$ & NA & $0.41 \pm 0.07$ & $0.54 \pm 0.00$ & $0.15 \pm 0.04$ & $0.09 \pm 0.01$ \\
\hline \multirow{4}{*}{$\begin{array}{c}\text { HDL } \\
(\mathrm{mmol} / \mathrm{l})\end{array}$} & 1 & $1.33 \pm 0.35$ & $1.62 \pm 0.29$ & $0.68 \pm 0.01$ & $1.53 \pm 0.41$ & $1.11 \pm 0.27$ & $1.72 \pm 0.86$ \\
\hline & 14 & $1.39 \pm 0.20$ & NA & $0.62 \pm 0.29$ & $1.71 \pm 0.00$ & $1.50 \pm 0.13$ & $1.23 \pm 0.08$ \\
\hline & 21 & $1.18 \pm 0.00$ & NA & $0.45 \pm 0.03$ & $1.32 \pm 0.23$ & $1.13 \pm 0.09$ & $0.90 \pm 0.11$ \\
\hline & 28 & $0.53 \pm 0.01$ & NA & $0.70 \pm 0.38$ & $2.86 \pm 0.00$ & $0.84 \pm 0.31$ & $0.85 \pm 0.18$ \\
\hline \multirow{4}{*}{$\begin{array}{c}\text { LDL } \\
(\mathrm{mmol} / \mathrm{l})\end{array}$} & 1 & $0.33 \pm 0.11$ & $0.84 \pm 0.60$ & $0.22 \pm 0.02$ & $0.59 \pm 0.41$ & $0.37 \pm 0.19$ & $0.52 \pm 0.11$ \\
\hline & 14 & $0.44 \pm 0.03^{\mathrm{a}}$ & NA & $0.28 \pm 0.60$ & $2.07 \pm 0.24$ & $0.80 \pm 0.72$ & $0.62 \pm 0.34$ \\
\hline & 21 & $0.94 \pm 0.00^{\mathrm{a}}$ & NA & $0.27 \pm 0.08$ & $1.41 \pm 0.61$ & $0.70 \pm 0.64$ & $0.73 \pm 0.13$ \\
\hline & 28 & $0.30 \pm 0.10^{\mathrm{d}}$ & NA & $0.23 \pm 0.05$ & $2.34 \pm 0.17$ & $0.21 \pm 0.06$ & $0.85 \pm 0.30$ \\
\hline \multirow{4}{*}{$\begin{array}{c}\mathrm{RBC} \\
\left(\mathrm{x} 10^{12} / \mathrm{l}\right)\end{array}$} & 1 & $5.30 \pm 0.06$ & $5.20 \pm 0.58$ & $5.05 \pm 0.56$ & $5.13 \pm 0.09$ & $5.40 \pm 0.04$ & $4.97 \pm 0.06$ \\
\hline & 14 & $5.60 \pm 0.04$ & NA & $5.47 \pm 1.17$ & $6.26 \pm 0.12$ & $5.23 \pm 0.22$ & $5.08 \pm 0.29$ \\
\hline & 21 & $5.92 \pm 0.01$ & NA & $5.80 \pm 0.43$ & $5.80 \pm 0.43$ & $4.65 \pm 0.95$ & $5.39 \pm 0.08$ \\
\hline & 28 & $6.65 \pm 0.29$ & NA & $5.57 \pm 1.11$ & $4.24 \pm 1.06$ & $4.68 \pm 0.02$ & $5.40 \pm 0.31$ \\
\hline \multirow{4}{*}{$\begin{array}{l}\text { WBC } \\
\left(\times 10^{9} / 1\right)\end{array}$} & 1 & $7.13 \pm 1.69$ & $8.70 \pm 0.95$ & $8.63 \pm 1.92$ & $8.67 \pm 0.72$ & $6.77 \pm 0.38$ & $7.37 \pm 0.95$ \\
\hline & 14 & $7.47 \pm 0.94$ & NA & $6.75 \pm 0.03$ & $8.90 \pm 0.06$ & $6.07 \pm 0.72$ & $8.97 \pm 1.03$ \\
\hline & 21 & $9.30 \pm 0.01$ & NA & $9.23 \pm 0.43$ & $9.20 \pm 0.43$ & $6.70 \pm 0.40$ & $8.52 \pm 0.76$ \\
\hline & 28 & $9.00 \pm 0.12$ & NA & $9.73 \pm 1.39$ & $10.87 \pm 0.89$ & $6.20 \pm 0.12$ & $8.30 \pm 0.46$ \\
\hline \multirow{4}{*}{$\begin{array}{l}\text { Platelet } \\
\left(\mathrm{x} 10^{9} / \mathrm{l}\right)\end{array}$} & 1 & $449.00 \pm 39.80$ & $391.00 \pm 23.03$ & $393.30 \pm 69.32$ & $383.67 \pm 45.06$ & $338.67 \pm 24.92$ & $371.00 \pm 63.11$ \\
\hline & 14 & $318.70 \pm 38.69^{c}$ & NA & $359.30 \pm 19.92$ & $282.70 \pm 0.88$ & $298.00 \pm 22.00$ & $269.70 \pm 20.49$ \\
\hline & 21 & $277.00 \pm 0.00^{c}$ & NA & $331.30 \pm 69.30$ & $301.30 \pm 69.30$ & $291.00 \pm 1.16$ & $252.00 \pm 4.62$ \\
\hline & 28 & $277.50 \pm 2.59$ & NA & $268.30 \pm 62.10$ & $204.50 \pm 28.58$ & $298.50 \pm 10.68$ & $246.50 \pm 3.75$ \\
\hline
\end{tabular}

Data represented as mean \pm SEM ( $\mathrm{n}=3$ for day 1,14 and $21 ; \mathrm{n}=2$ for day 28 ), $\mathrm{NA}=$ mean value not available as 2 of the 3 female rabbits in group1 died within a week of the study, superscript a = significant increase on days 14 and 21 over day 1 ( $\mathrm{p}<0.05$ by ANOVA), superscript $\mathrm{c}=$ significant decrease on days 14 and 21 over day 1 ( $p<0.05$ by ANOVA), superscript $d=$ significant decrease on 


\section{Macrothink}

day 28 over day $21(\mathrm{p}<0.05$ by Student's $\mathrm{t}$ test $), \mathrm{TC}=$ Total Cholesterol, $\mathrm{TG}=$ Triglyceride, $\mathrm{HDL}=$ High Density Lipoprotein, LDL $=$ Low Density Lipoprotein, $\mathrm{RBC}=$ Red Blood Cell, $\mathrm{WBC}=$ White Blood Cell, $\mathrm{M}=$ Male, $\mathrm{F}=$ Female, $\mathrm{SEM}=$ Standard Error of Mean

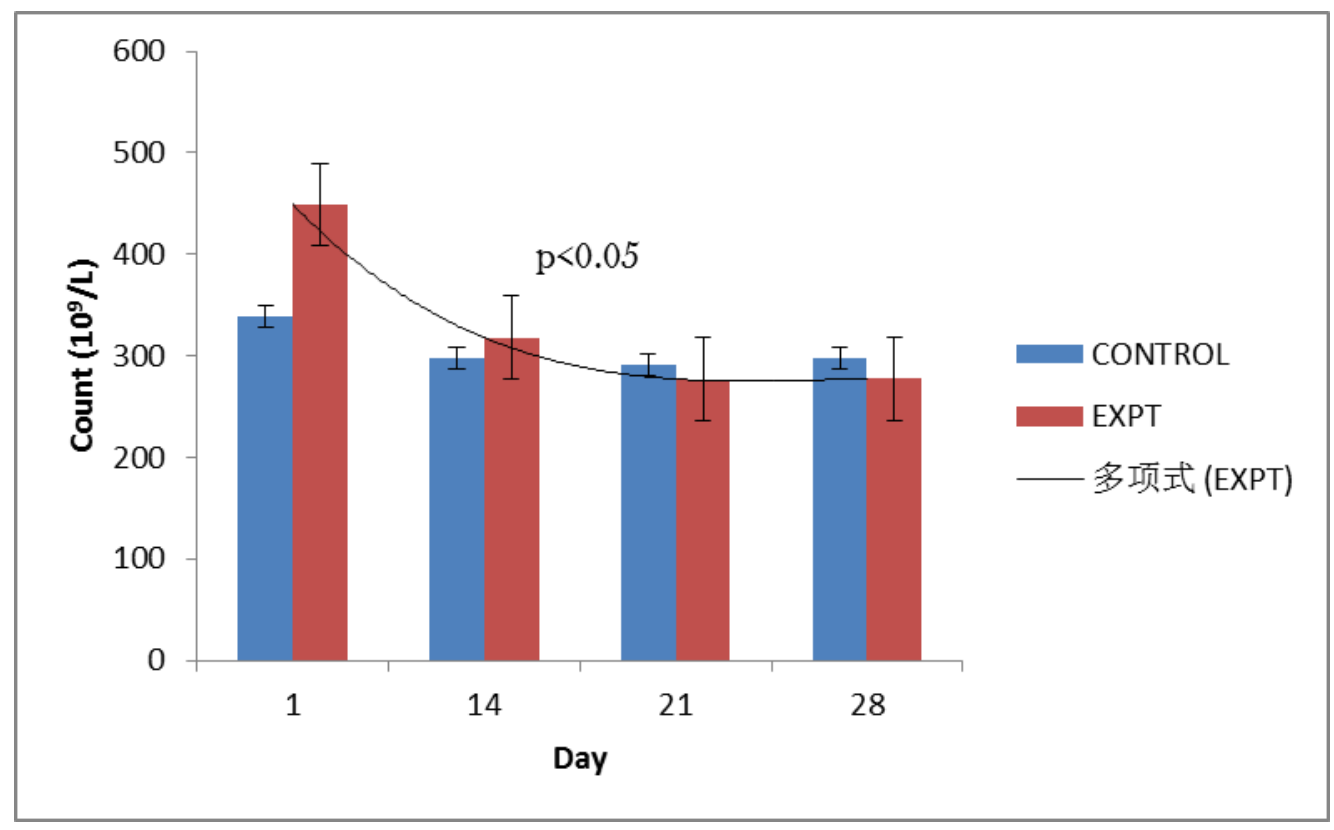

Fig 3: Effects of $23.1 \mathrm{ml} \mathrm{RB}$ drink on platelet count of group 1 male rabbits

\section{Effects of $R B$ drink on histology of rabbits}

The hepatic system of rabbits in group 1 that received $23.1 \mathrm{ml}$ of $\mathrm{RB}$ drink showed congestion of the central vein of the liver (Fig 4) but that of rabbits in groups 2 and 3 showed normal organs architecture (Fig 5)

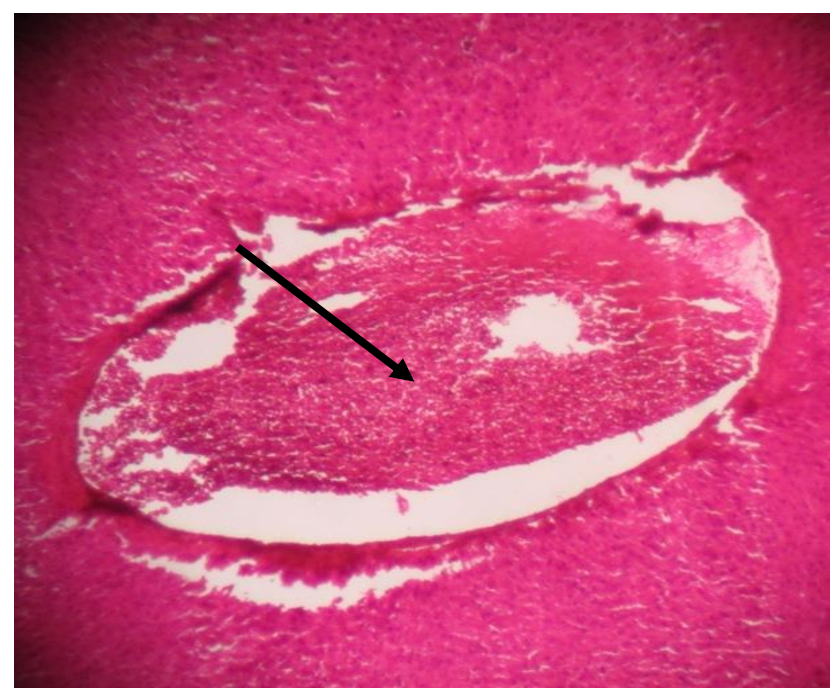

Fig 4: Histology of hepatocyte with congestion of central vein of representative rabbits in group 1 (1000 x magnification) 


\section{MInstitute ${ }^{\text {Macrothink }}$}

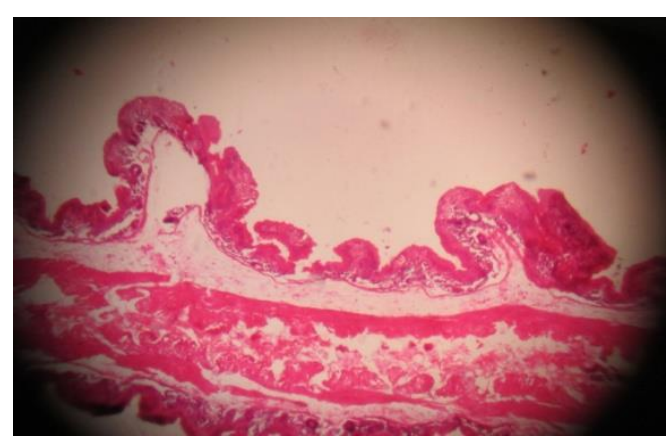

Intestine

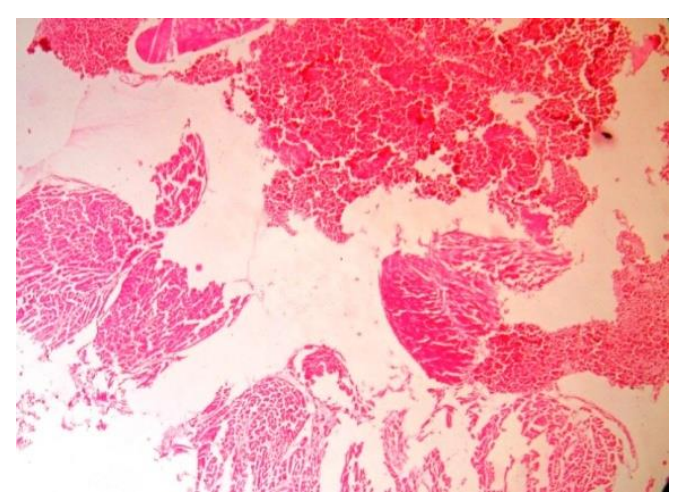

Heart

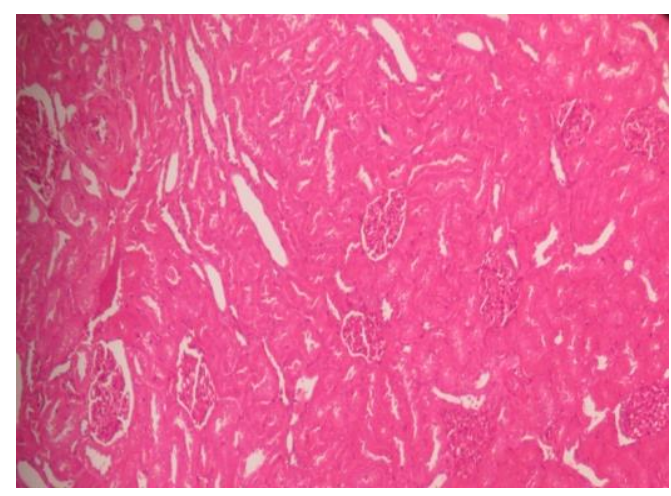

Kidney

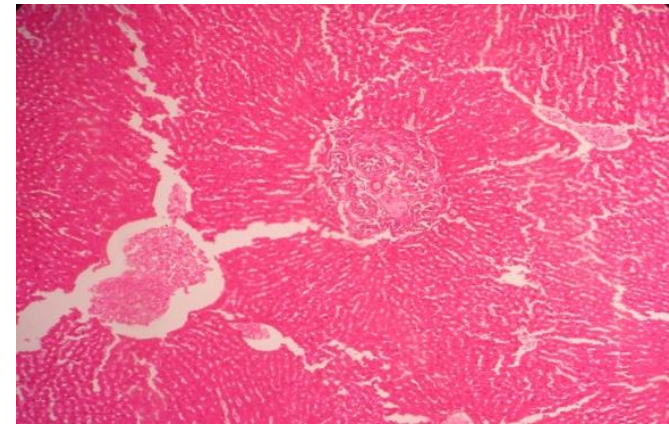

Liver

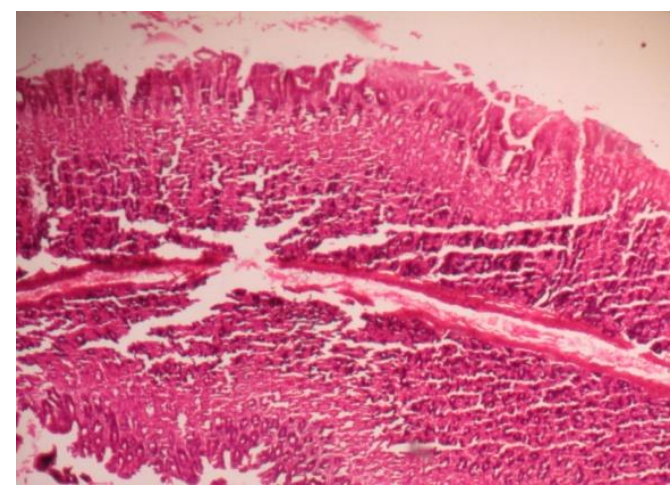

Stomach

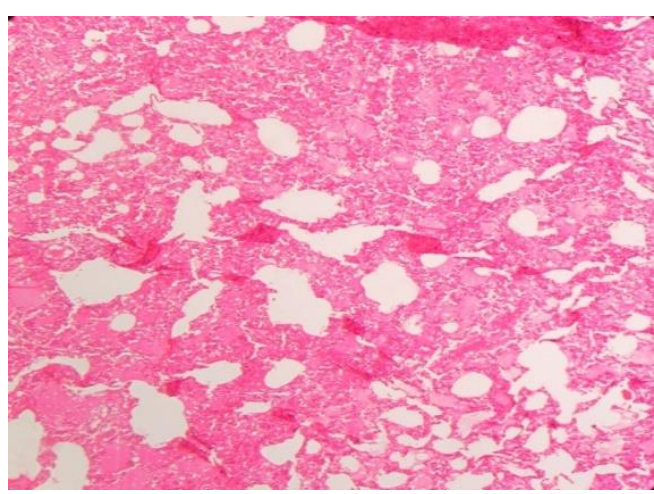

Lung

Fig 5: Histology of organs of representative rabbits in groups $2 \& 3$ showing normal architecture (1000x magnification) 


\section{Discussion}

Safety concern over the consumption of energy drinks has continued to be raised by international bodies such as Scientific Committee on Food (SCF) of the European Food Safety Authority (SCF, 1999; SCF, 2003; EFSA 2009). In the United States, a number of stimulant energy drinks were withdrawn from markets based on federal legislation which mandated manufacturers to prove that all ingredients/constituents in the drinks are safe for consumption, even if they have received prior approval for use. The manufacturers of the products involved were then mandated to produce scientific evidence to the Food and Drug Administration (FDA) that their products are safe (FDA, 2001).

In this study, the experimental group that received $23.1 \mathrm{ml}$ of RB drink daily (group 1), which is equivalent to a $60 \mathrm{~kg}$ adult consuming one can or $250 \mathrm{ml}$ of a typical energy drink daily, the rabbits (both sexes) when compared with the control, consistently exhibited increased activity within 1 hour of intake after which they became calm. This observation is consistent with reports of similar studies of RB conducted on rats (Red Bull GmbH, 2000). The behavioural changes in the rat studies by the Red Bull company have been attributed to the central pharmacological effect of taurine constituent of the energy drink, but this effect is usually observed at high taurine dose of $1000 \mathrm{mg} / \mathrm{kg}$ body weight (WIL, 2001a). This contradicts what is observed in this present study, as the dose of taurine in $23.1 \mathrm{ml}$ of RB drink, which is $92.6 \mathrm{mg}\left(51.42 \mathrm{mg} / \mathrm{kg}\right.$ body weight), is far lower $\left(1 / 19^{\text {th }}\right)$ than the dose administered in the Red Bull company study on rats.

The other possibility to consider for the rabbit behaviour in this group is the caffeine content in the energy drink. Studies conducted in humans have shown that a single serving of caffeinated beverage containing $40-120 \mathrm{mg}(0.65 \mathrm{mg} / \mathrm{kg}-1.95 \mathrm{mg} / \mathrm{kg})$ of caffeine produced a stimulatory biological effects and promoted alertness, enhanced cognitive performance, relieved fatigue and promoted physical endurance (Doherty and Smith, 2004; Lorists and Top, 2003; Astorino and Robertson, 2010). This single serving can also cause transient adverse effects such as insomnia, headaches and nervousness in caffeine-sensitive individuals (Nawrot et al., 2003; Higdon and Frei, 2006). In this rabbit study, the dose of caffeine in 23.1 $\mathrm{ml}$ of $\mathrm{RB}$ is $7.2 \mathrm{mg}$, which is equivalent of $80 \mathrm{mg}$ caffeine in one can of RB consumed by an adult weighing $60 \mathrm{~kg}$. Although metabolism of caffeine in rabbits may be slightly different from human, the fact remains that caffeine content of $\mathrm{RB}$ drink is most likely factor responsible for increased behavioural activity of the animals through its effects on the brain (Fisone et al., 2004).

There were observed reversible changes in some biochemical parameters of rabbits in this group especially with the male rabbits. Total proteins (TP) and liver enzymes activity especially alanine transaminase (ALT) and alkaline phosphatase (ALP) were significantly increased in male rabbits on days 14 and 21 of the study, when compared with the baseline. Transaminases (ALT and AST) play an important role in protein and amino acid metabolism which are found in the cells of almost all the body tissues, and when diseases or injuries affect these tissues, they are released into the blood stream (Kuzminskaya and Bersan, 1975). Serum ALT activity is known to increase when there is hepatic parenchyma damage, and it 
has been employed as a tool for measuring hepatic necrosis (Kaplan, 1986). The other transaminase, AST, which activity is usually increased alongside ALT in hepatocellular injury, was however not significantly elevated on days 14 and 21 in this study. AST, which is both cytosolic and mitochondrial in location, is not a liver specific enzyme as it can also be found in skeletal and cardiac muscles as well as in the red blood cells (Etuk and Mohammed, 2010).

Alkaline phosphatase (ALP) activity was however significantly raised in the plasma and this increase may be considered as an indicator of cholestasis that may result from intracellular hepatic canaliculi obstruction associated with inflammation (Birkner et al., 2006). However, plasma ALP activity like AST may also be affected by sources in the body other than the liver, limiting its use as a marker of hepatic dysfunction. This was the case with female rabbits in our control group which showed slight increase in ALP activity despite not receiving energy drink. One haematological parameter that was significantly reduced on days 14 and 21 is platelet, and this may suggest some degree of haematopoietic injury.

All these findings could not be confirmed in the female rabbits in this group as 2 of the 3 rabbits died within the first week of the study, which made sampling on days 14, 21 and 28 impossible. Nevertheless, the findings in the male rabbits in our study agrees with some of the findings of the 13 week oral sub-chronic toxicity study of mice (Red Bull GmbH, 2000) administered $0 \%, 33 \%, 50 \%$ and $100 \% \mathrm{RB}$ concentrations, in which there was a significant increase in ALP activity but decrease in platelet count among male mice given 100\% RB. From the mice studies (Red Bull GmbH, 2000; EFSA, 2009), it was concluded that most of the statistically significant findings in haematology and clinical chemistry may be attributable to increased fluid intakes and glucose loading from sucrose in RB drink.

However, one recent 28-day sub-acute toxicodynamic study tested another energy drink "Power Horse" on hepatic, renal and histological functions of Sprague-Dawley rats (Akande and Banjoko, 2011) and reported reversible increase in ALT and ALP activity, and urea and some electrolytes concentration, which agrees with findings of the current study on rabbits. From the findings of this current study and some more recent ones testing different types of energy drink on rats (Khayyat et al., 2012; Chimezie, 2013; Khayyat et al., 2014a, 2014b) and rabbits (Ebuehi et al., 2011), RB and other energy drinks may be associated with acute reversible hepatic, renal and possibly haematopoietic injury, which may not be totally explained by increased fluid intake or glucose loading in the drink. Furthermore, the histology of one female rabbit in this experimental group that died within the first week of the study and male rabbits sacrificed on day 28 showed hepatic abnormalities such as congestion of central veins and balloon degeneration of hepatocytes. Similar hepatic and renal pathologies were reported in rats by many other researchers (Akande and Banjoko, 2011; Khayyat et al., 2012; Khayyat et al., 2014a), lending credence to the fact that injuries caused by RB and other energy drinks cannot solely be explained by increased fluid intake. In the group of rabbits (group 2) that received $11.5 \mathrm{ml}$ of $\mathrm{RB}$, a dose equivalent to half can or $125 \mathrm{ml}$ of energy drink (which contains $46.3 \mathrm{mg}$ taurine and $3.6 \mathrm{mg}$ caffeine), there were no significant biochemical or haematological changes in the male rabbits and no mortality was recorded. However in the female rabbits, significant increase in ALT activity, and in levels of total proteins occurred while serum albumin levels decreased, but these parameters reverted 
on withdrawal of the RB drink. Rabbits in this group equally exhibited consistently increased behavioural activity after intake of the drink daily. The biochemical and haematological changes observed at this dose of RB have been previously reported in rats (Red Bull GmbH, 2000; Akande and Banjoko, 2011). These changes including the rabbit behaviour can also be attributed mainly to the caffeine content of the RB. The caffeine content of this dose of RB (3.6 mg) is equivalent to $40 \mathrm{mg}$ caffeine intake in an adult human, which can still produce stimulatory biological effects and increased activities (Doherty and Smith, 2004; Lorists and Top, 2003; Astorino and Robertson, 2010).

Results of this study also showed female rabbits to be more adversely affected by intake of the energy drink, as mortality was recorded in group 1, and female rabbits in group 2 had more deranged biochemical parameters than the male rabbits. The reason for this may not be obvious but hormonal differences between males and females may be a plausible explanation. From the results of this study, it can be concluded that consumption of RB energy drink is associated with adverse biochemical, haematological and histopathologic effects on rabbits at daily intake of $23.1 \mathrm{ml}$ (equivalent to an adult human drinking one can or $250 \mathrm{ml}$ of RB daily) and at lower dose of $11.5 \mathrm{ml}$ (equivalent to an adult human drinking half can or $125 \mathrm{ml}$ of RB daily). These doses are lower than the daily intake amount of 2 cans $(500 \mathrm{ml})$ recommended by the manufacturer of RB energy drink (Red Bull $\left.{ }^{(\mathrm{R})} \mathrm{GmbH}\right)$.

The consumption of RB energy drink must be done with caution. There is also the need for the Federal Government of Nigeria to; (1) regularly assess the safety of the many types of energy drinks in Nigeria through laboratory testing; (2) conduct intake study of young adults and children about consumption of energy drink, and (3) regulate the sale of many of the energy drinks through legislation by appropriate agency of Government such as the National Agency for Food and Drug Administration and Control

\section{Acknowledgement}

The contribution of Consultant Histopathologist and Laboratory Scientists in the College of Health Sciences, Ladoke Akintola University of Technology, Osogbo, Nigeria is highly appreciated. 


\section{MInstitute ${ }_{\text {Mnk }}^{\text {Macrothin }}$}

\section{References}

Akande IS, Banjoko OA. (2011). Assessment of biochemical effect of "Power Horse" energy drink on hepatic, renal and histological functions in Sprague-Dawley rats. Ann. Rev. Res. Biol. 1(3): $45-56$

Arneson W, Brickell J. (2007). Clinical Chemistry: A Laboratory Perspective. FA Davis Co., Philadelphia. pp. $201-266$

Astorino TA, Robertson DW. (2010). Efficacy of acute caffeine ingestion for short-term high-intensity exercise performance: a systematic review. Journal of Strength Condition Research. 24(1): 257-265

Australian and New Zealand Food Standard Council (ANZFSC) (2000) Full Assessment report and regulation impact assessment. Application A394: Formulated caffeinated beverages. URL:http://www.anzfa.gov.au/_srcfiles/A394_(full)_report.pdf (accessed November 2015)

Birkner E, Grucka-Mamczar E, Zwirska-Korczala K, Zalejska-Foilka J, Starwiarska-Pieta B, Kasperczyk S, Kasperczyk A. (2006). Influence of sodium fluoride and caffeine on the kidney function and free-radical processes in that organ in adult rats. Biology and Trace Element Research. 112: 169 - 174

Bowers GN Jr, McComb RB. (1966). A continuous spectrophotometric method for measuring the activity of serum alkaline phosphatase. Clinical Chemistry. 12: $70-89$

Chimezie OS. (2013). Effects of Bullet Energy Drink on Creatinine phosphokinase (CPK) and Lactate Dehydrogenase (LDH) Level of Albino Rat. Journal of Natural Sciences Research.3(3): 15-17

Doherty M, Smith PM. (2004). Effects of caffeine ingestion on exercise testing: a metaanalysis. International Journal of Sport Nutrition, Exercise and Metabolism. 14: 626-646

Ebuehi OA, Ajayi OE, Onyculor AI, Awelimobor D. (2011). Effects of oral administration of energy drinks on blood chemistry, tissue histology and brain acetylcholine in rabbits. Nigerian Quarterly Journal of Hospital Medicine. 21(1): 29 - 34

Etuk EU, Mohammed AA. (2010). Safety evaluations of aqueous stem bark extract of Lophira lanceolata in Sprague-Dawley rats. International Journal of Research and Pharmaceutical Sciences. 1: 28 - 33

European Food Safety Authority (EFSA). (2009). Scientific Opinion of the Panel on Food Additives and Nutrient Sources added to Food on a request from the Commission on the use of taurine and D-glucurono- $\gamma$-lactone as constituents of the so called "energy" drinks. The EFSA Journal. 935: 1-31.

Fisone GG, Borgkvist A, Usiello A. (2004). Caffeine as a psychomotor stimulant: mechanism of action. Cellular and Molecular Life Science. 61(7-8): 857-872. 


\section{I Macrothink}

Journal of Biology and Life Science ISSN 2157-6076 2018, Vol. 9, No. 1

Food and Drug Administration (FDA) (2001). Statement on the use of herbals in foods. URL:http://www.cfsan.fda.gov/ dms/dsltr15. html (Accessed Nov 2015)

Food Safety and Protection Board (FSPB). (2003). A review of health effects of stimulant drinks, 2003, Ireland.

http://www.safefood.eu/Global/Publications/Research\%20reports/FSBP\%20Stimulant\%20dri nks\%20.pdf?epslanguage $=$ en $($ Accessed November 2015)

Higdon JV, Frei B. (2006). Coffee and health: a review of recent human research. Critical Reviews in Food Science and Nutrition. 46: 101-123

Ikegwuonu FI, Aire TA, Ogwuegbu SO. (1981). Effect of kolanut extract administration on the liver, kidney, brain, testes and some serum constituents of rats. Journal of Applied Toxicology. 1: $292-294$

Kaplan MM. (1986). Serum alkaline phosphatase - another piece is added to the puzzle. Hepatology. 6: 526

Khayyat L, Sorour J, Al Rawi M, Essawy A. (2012). Histological, ultrastructural and physiological studies on the effect of different kinds of energy drinks on the liver of Wistar albino rat. Journal of American Science. 8(8): 688 - 697

Khayyat L, Essawy A, Sorour J, Al Rawi M. (2014a). Impact of some energy drinks on the structure and function of the kidney in Wistar albino rats. Life Science Journal. 11(10): 1131 1138

Khayyat L, Essawy A, Al Rawi M, Sorour, J. (2014b). Detrimental effects of energy drink on haematopoietic system in rats. Journal of Environmental Biology. 35: 883 - 891

Kuzminskaya UA, Bersan LV. (1975). The effects of the sodium salt of dichlorophenoxy-acetic acid on the glycolysis. ATPase and transketolase activity of erythrocytes. Journal Farmakol Toksikol. 38: 102 - 104

Lorist M, Tops MM. (2003). Caffeine, fatigue and cognition. Brain Cognition. 53: 82-94

Luna LC. (1968). Manual of histologic staining methods. Armed Forces Institute of Pathology. $3^{\text {rd }}$ edn, New York, McGraw Hill Book Company

Nawrot P, Jordan S, Eastwood J. (2003). Effects of caffeine on human health. Food Additives and Contaminants. 20(1): 1-30

OECD. (2008). OECD Guidelines for testing of chemicals. Repeated 28-day oral toxicity study in rodents. Guideline 407. Organization for Economic Co-operation and Development, Paris.

Rallis D. (2001). Cases associated with the consumption of Red Bull. Letter to the Director-General of DG SANCO from Greek Permanent Representation to the European Union. Brussels, $12^{\text {th }}$ October 2001

$\operatorname{Red~Bull}^{(\mathrm{R})}(\mathrm{GmbH})$, Brunn 115, A-5330 Fuschl am See, Austria 


\section{Macrothink}

Red Bull GmbH (2000). Red Bull: 13 week oral toxicity (drinking water) study in the mouse. Final Report. Authors: Schmid H, Richard D, Luetkemeier H, Biedermann K and Millar PM. RCC project 719144, RCC, Switzerland. Submitted to the European Commission by the study sponsor, Red Bull GmbH, Brunn 115, A-5330 Fuschl am See, Austria.

Reinhold JG. (1953). In: Reiner M (ed). Standard Methods of Clinical Chemistry, New York and London, Academic Press. pp. 88

Rotstein J, Barber J, Strowbridge C, Hayward S, Huang R, Godefroy SB. (2013). Energy drinks: an assessment of the potential health risks in the Canadian context. International Food Risk Analysis Journal. 3(5): 1 - 29.

SCF (Scientific Committee for Food). (1999). Opinion on caffeine, taurine and D-glucurono- $\gamma$-lactone as constituents of so-called 'energy' drinks.

URL:http://europa.eu.int/comm/food/fs/sc/scf/out 22_en.html (Accessed Nov 2015)

SCF (Scientific Committee on Food Authority). (2003). Opinion of Scientific Committee of Food on Additional Information on 'energy' drink (expressed on 5 March 2003) European Commission, Brussels

Searcy RL, Reardon JE, Foreman JA. (1967). Urea determination. American Journal of Tropical Medicine and Technology. 33: 15 - 20

Shin J, Seol I, Son C. (2010). Interpretation of animal dose and human equivalent dose for drug development. Journal of Korean Oriental Medicine. 31(3): 1 - 7

Stimulant Drink Committee. (2002). A review of health effects of stimulant drinks. Final Report. Commissioned by the Food and Safety Promotion Board, Ireland. Available at www.safefoodonline.com/pdf/health_effects_of_stimulant_drink_pdf (Accessed June 2014)

Tietz NW. (2000). Fundamental of Clinical Chemistry. London, W. B. Saunders Company. pp $1020-1038$

United States Food and Drug Administration (USFDA). (2005). Guideline for Industry. Estimating the maximum safe starting dose in initial clinical trials for therapeutics in adult healthy volunteers. Centre for Drug Evaluation and Research, US Department of Health and Human Services http://www.fda.gov/cder/guidance/index.htm (Accessed June 2014)

WIL (2001a). A 13-week oral (gavage) toxicity study of taurine in rats. Final Report, December 26, 2001, WIL-423002. WIL Research Laboratories Inc., Ohio, USA. Submitted to the European Commission by Red Bull GmbH, Brunn 115, A-5330 Fuschl am See, Austria, 31 December 2001. 


\section{Copyright Disclaimer}

Copyright for this article is retained by the author(s), with first publication rights granted to the journal.

This is an open-access article distributed under the terms and conditions of the Creative Commons Attribution license (http://creativecommons.org/licenses/by/3.0/). 